\title{
A note on chiral trace relations from qq-characters
}

\author{
Saebyeok Jeong ${ }^{a, b}$ and Xinyu Zhang ${ }^{b}$ \\ ${ }^{a}$ C.N. Yang Institute for Theoretical Physics, Stony Brook University, \\ Stony Brook, NY 11794-3840, U.S.A. \\ ${ }^{b}$ NHETC and Department of Physics and Astronomy, Rutgers University, \\ Piscataway, NJ 08854, U.S.A. \\ E-mail: saebyeok.jeong@physics.rutgers.edu, \\ zhangxinyuphysics@gmail.com
}

ABSTRACT: We study chiral operators in four-dimensional $\mathcal{N}=2$ supersymmetric gauge theories. We provide a general procedure to derive the exact relations among the vacuum expectation values of chiral operators in the $\Omega$-background using the non-perturbative Dyson-Schwinger equations of qq-characters. We demonstrate our procedure using several examples. For pure $\mathrm{SU}(N)$ gauge theory and $\mathrm{SU}(N)$ gauge theory with $2 N$ fundamental hypermultiplets, we verify the relations conjectured previously in the literature. We also briefly discuss the relations in $\mathrm{SU}(N)$ linear superconformal quiver gauge theories.

KEYWORDS: Extended Supersymmetry, Nonperturbative Effects, Supersymmetric Gauge Theory

ARXIV EPRINT: 1910.10864 


\section{Contents}

1 Introduction 1

2 General strategy $\quad 4$

2.1 Chiral ring relations on $\mathbb{R}^{4} \quad 4$

2.2 Chiral trace relations in the $\Omega$-background 5

3 Basic examples of SU $(N)$ gauge theories $\quad 7$

3.1 Pure $\mathrm{SU}(N)$ theory $\quad 7$

$\begin{array}{lll}3.1 .1 & \text { Partition function and qq-characters } & 7\end{array}$

$\begin{array}{ll}3.1 .2 & \text { Chiral trace relations }\end{array}$

$\begin{array}{lll}3.2 & \mathrm{SU}(N) \text { theory with } 2 N \text { fundamental hypermultiplets } & 10\end{array}$

$\begin{array}{lll}3.2 .1 & \text { Partition function and qq-characters } & 10\end{array}$

$\begin{array}{lll}3.2 .2 & \text { Chiral trace relations } & 11\end{array}$

4 The linear quiver gauge theory $\quad 11$

\section{Introduction}

Ever since the pioneering work of Seiberg and Witten [1, 2], four-dimensional $\mathcal{N}=2$ supersymmetric gauge theories have been repeatedly investigated from various viewpoints. Both the low energy effective prepotential $\mathcal{F}_{\mathrm{SW}}$ and the correlation functions of $\mathcal{N}=2$ chiral operators can be determined in terms of period integrals of the Seiberg-Witten meromorphic differential $\lambda$ on the Seiberg-Witten curve $\Sigma$.

In order to provide a rigorous derivation of the Seiberg-Witten solution, it is useful to put the theory in the $\Omega$-background [3, 4], which effectively regularizes the infinite volume of the spacetime $\mathbb{R}^{4}$ while preserving a part of the deformed supersymmetry. The partition function $\mathcal{Z}$ in the $\Omega$-background can be computed exactly using the localization technique for a large class of traditional Lagrangian theories constructed using the vector multiplet with gauge group $G$ and the hypermultiplet in the representation $\mathfrak{R}$ of $G$. When the gauge group is a unitary group, the result can be written as a statistical sum over a collection of Young diagrams $\vec{\lambda}$ labeling the special instanton configurations [4],

$$
\mathcal{Z}\left(\vec{a}, \vec{m}, \mathrm{q} ; \varepsilon_{1}, \varepsilon_{2}\right)=\sum_{\vec{\lambda}} \mu_{\vec{\lambda}}
$$

where $\vec{a}, \vec{m}, \mathrm{q},\left(\varepsilon_{1}, \varepsilon_{2}\right)$ are the Coulomb branch parameters, the masses of the hypermultiplets, the instanton counting parameter, and the $\Omega$-deformation parameters, respectively. The measure factor $\mu_{\vec{\lambda}}$ contains the classical, the one-loop and the instanton contributions 
from both the vector multiplet and the hypermultiplet. In the refined genus expansion of the partition function around the flat space limit $\varepsilon_{1}, \varepsilon_{2} \rightarrow 0$,

$$
\log \mathcal{Z}=-\sum_{g, n=0}^{\infty}\left(\varepsilon_{1} \varepsilon_{2}\right)^{g-1}\left(\varepsilon_{1}+\varepsilon_{2}\right)^{n} \mathcal{F}_{\left(g, \frac{n}{2}\right)},
$$

the leading term $\mathcal{F}_{(0,0)}$ coincides with the low energy effective prepotential $\mathcal{F}_{\mathrm{SW}}$ of the theory on $\mathbb{R}^{4}$. Hence we can derive the Seiberg-Witten geometry directly for a large class of $\mathcal{N}=2$ theories [5-9]. The higher order terms in the expansion (1.2) compute the couplings of the theory to the background gravitational field $[4,5]$, and are naturally linked with topological strings on non-compact Calabi-Yau threefolds.

Another interesting relation between the partition function in the $\Omega$-background and the disconnected partition function of A-model topological strings on Riemann surfaces was discovered in [10] and later generalized in [11-13]. The higher Casimir operators in the gauge theory are mapped to gravitational descendants of the Kahler form in the topological string theory.

It was conjectured in $[4,10]$ that the partition function in the $\Omega$-background should be related to certain two-dimensional conformal field theories or deformations thereof. A concrete realization of this conjecture was found between the four-dimensional $\mathcal{N}=2$ theory constructed by compactifying the six-dimensional $(2,0)$ theory on a punctured Riemann surface $\mathcal{C}[14,15]$ and two-dimensional Liouville/Toda theory living on $\mathcal{C}[16,17]$. Meanwhile, if we take the limit $\varepsilon_{1} \rightarrow 0$ and keep $\varepsilon_{2}=\hbar$ fixed so that the $\Omega$-background preserves two-dimensional $\mathcal{N}=2$ super-Poincare invariance, we get a quantization of the classical algebraic integrable systems underlying the four-dimensional $\mathcal{N}=2$ theories [18-20].

Therefore, we have sufficient reasons to carefully explore all the consequences of the generic $\Omega$-background. In this paper, we focus on the $\mathcal{N}=2$ chiral ring, which is believed to be freely generated on $\mathbb{R}^{4}$. Hence, we can choose a finite-dimensional basis of chiral operators such that any element of the chiral ring can be represented uniquely as a polynomial in the basis elements. The number of generators of the chiral ring is the complex dimension of the Coulomb branch, and the polynomial equations representing generic chiral operators in terms of the chosen basis elements are called chiral ring relations. For simple Lagrangian theories on $\mathbb{R}^{4}$, the chiral ring relations were derived in [21, 22]. It is interesting to ask what happens when we introduce the $\Omega$-deformation. The vacuum expectation value of a chiral operator $\mathcal{O}$ in the $\Omega$-background is given by $[10,11]$

$$
\langle\mathcal{O}\rangle=\frac{1}{\mathcal{Z}} \sum_{\vec{\lambda}} \mu_{\vec{\lambda}} \mathcal{O}_{\vec{\lambda}}
$$

where $\mathcal{O}_{\vec{\lambda}}$ is the value of $\mathcal{O}$ evaluated at $\vec{\lambda}$, and the vacuum expectation value of $\mathcal{O}$ on $\mathbb{R}^{4}$ can be obtained by taking the flat space limit,

$$
\langle\mathcal{O}\rangle_{\mathbb{R}^{4}}=\lim _{\varepsilon_{1}, \varepsilon_{2} \rightarrow 0}\langle\mathcal{O}\rangle
$$

For simplicity, we adopt the terminology used in [23, 24], and refer to the equations representing the vacuum expectation values of generic chiral operators in terms of the vacuum 
expectation values of the chosen basis elements as chiral trace relations. On $\mathbb{R}^{4}$, the chiral trace relations contain the same amount of information as the chiral ring relations due to the property of the cluster decomposition (see (2.2)). However, they are not equivalent once we introduce the $\Omega$-deformation, since the $\Omega$-background breaks the translational symmetry of $\mathbb{R}^{4}$. A priori it is not guaranteed that chiral trace relations exist in the general $\Omega$ background. Even if they exist, they need not to be polynomial equations. Regardless, the chiral operators are conjectured to be mapped to the integrals of motion in two-dimensional conformal field theories $[16,25,26]$. From the considerations in the conformal field theory, we expect such chiral trace relations in the $\Omega$-background still exist. Indeed, several groups recently computed the vacuum expectation values of chiral operators in the $\Omega$-background for some simple theories, and conjectured the chiral trace relations in the $\Omega$-background based on the explicit series expansion in $\mathrm{q}[23,24,27,28]$. The problem is that neither the brute force method nor the analysis based on two-dimensional conformal field theory can be easily generalized to gauge theories with gauge group of high rank or with general matter content.

The purpose of this note is to provide a more systematic field theoretical derivation of the chiral trace relations in the $\Omega$-background. We will show that the non-perturbative Dyson-Schwinger equations, which follow from the regularity of the vacuum expectation values of qq-characters [29], give a simple framework for investigating the chiral trace relations. Our approach can be regarded as the natural counterpart of the derivation of the classical chiral ring relations on $\mathbb{R}^{4}$. We will work out the details in the pure $\mathrm{SU}(N)$ gauge theory and the $\mathrm{SU}(N)$ gauge theory with $2 N$ fundamental hypermultiplets to illustrate our strategy, and then generalize to the linear $\mathrm{SU}(N)$ superconformal quiver gauge theories. We find that the chiral trace relations in the $\Omega$-background can dramatically modify the relations on $\mathbb{R}^{4}$. Similar strategy was also used in the derivation of the BPZ equations from the point of view of four-dimensional $\mathcal{N}=2$ supersymmetric gauge theories [30, 31].

It is possible to generalize our approach to other $\mathcal{N}=2$ theories if their qq-characters are known. For example, it is a good exercise to derive the chiral trace relations in the $\Omega$ background for the $\mathcal{N}=2^{*}$ theory, and check the conjectures made in [23, 24, 28]. It is also interesting to introduce half-BPS surface defects. There are several different approaches to introduce surface operators in the $\Omega$-background [32], and it is straightforward to derive the chiral trace relations for all these approaches. Some of the cases have already been studied in [33] in the context of their relation to the symplectic geometry of the moduli space of flat connections on a Riemann surface. Also, it was discovered in [34] that the surface defect partition functions split into parts at special loci of the moduli space, accounting for the splitting of degenerate levels in the corresponding quantum integrable system. It would be interesting to study how the chiral trace relations are affected at those loci. In [35], the gauge theory partition functions were reconstructed in terms of representations of the quantum toroidal algebra of $\mathfrak{g l}(1)$. The reconstruction was further extended to the surface defect partition functions in [36], with a proper generalization of the relevant quantum algebra. It would be nice to investigate the algebraic meaning of the chiral trace relations discussed in this note in this algebraic engineering context. Another interesting problem is to consider the qq-characters when we put the theory on compact spaces such as (squashed) four-spheres [37, 38]. 
The rest of the paper is organized as follows. In section 2, we outline our general strategy to derive the chiral trace relations in the $\Omega$-background. In section 3 , we show explicitly how to obtain the chiral trace relations in the $\Omega$-background in the pure $\mathrm{SU}(N)$ gauge theory and the $\mathrm{SU}(N)$ gauge theory with $2 N$ fundamental hypermultiplets. In section 4 , we generalize our discussion to the linear $\mathrm{SU}(N)$ superconformal quiver gauge theories.

\section{General strategy}

\subsection{Chiral ring relations on $\mathbb{R}^{4}$}

In four-dimensional $\mathcal{N}=2$ supersymmetric gauge theories, a chiral operator $\mathcal{O}(x)$ is defined to be a gauge-invariant local operator annihilated by the action of all supercharges $\bar{Q}_{\dot{\alpha} A}$ of one chirality,

$$
\left[\bar{Q}_{\dot{\alpha} A}, \mathcal{O}(x)\right]=0 .
$$

Given such an operator, one can always generate its superpartners by acting on it with the $Q_{\alpha A}$. It follows immediately from the $\mathcal{N}=2$ supersymmetry algebra on $\mathbb{R}^{4}$ and the Jacobi identity that a product of chiral operators is still chiral, and its vacuum expectation value is independent of their spacetime positions. Then one can take the limit of large separation and apply the cluster decomposition to factorize correlation functions of chiral operators,

$$
\left\langle\mathcal{O}_{1}\left(x_{1}\right) \mathcal{O}_{2}\left(x_{2}\right) \cdots \mathcal{O}_{n}\left(x_{n}\right)\right\rangle=\left\langle\mathcal{O}_{1}\right\rangle\left\langle\mathcal{O}_{2}\right\rangle \cdots\left\langle\mathcal{O}_{n}\right\rangle
$$

where on the right hand side we no longer need to specify the positions.

Since $\bar{Q}_{\dot{\alpha} A}$-exact objects decouple in the vacuum expectation values, we can define an equivalence relation between two chiral operators $\mathcal{O}_{1}\left(x_{1}\right)$ and $\mathcal{O}_{2}\left(x_{2}\right)$ if there exist a gauge invariant operator $X$ such that

$$
\mathcal{O}_{1}\left(x_{1}\right)=\mathcal{O}_{2}\left(x_{2}\right)+\left[\bar{Q}_{\dot{\alpha} A}, X\right]
$$

The set of equivalence classes of chiral operators forms a commutative ring, known as the chiral ring [21]. We refer to the generators of the chiral ring as basis chiral operators, whose number is the complex dimension of the Coulomb branch. Any element of the chiral ring can be represented uniquely in terms of the basis element. We call the set of all such expressions the chiral ring relations. The chiral ring is completely specified by the set of basis chiral operators and the chiral ring relations.

At the classical level, the chiral ring relations follow from group theoretical identities. In this note, we focus on the $\mathrm{SU}(N)$ gauge theory with arbitrary matter. Let $\Phi$ be the adjoint scalar field in the vector multiplet. We can choose the basis chiral operators to be

$$
\mathcal{O}_{n}=\frac{1}{n} \operatorname{Tr} \Phi^{n}, \quad n=2, \cdots, N
$$

whose vacuum expectation values parametrize the Coulomb branch of moduli space of vacua. Since $\Phi$ is a traceless $N \times N$ matrix, we can solve the Coulomb moduli $\vec{a}=\left(a_{1}, \cdots, a_{N}\right)$ 
in terms of $\mathcal{O}_{n}, n=2, \cdots, N$. Any other chiral operator can be expressed in terms of $\vec{a}$. For example,

$$
\mathcal{O}_{n_{1}, n_{2}, \cdots, n_{\ell}}=\prod_{i=1}^{\ell}\left(\frac{1}{n_{i}} \operatorname{Tr} \Phi^{n_{i}}\right)=\prod_{i=1}^{\ell}\left(\frac{1}{n_{i}} \sum_{\alpha=1}^{N} a_{\alpha}^{n_{i}}\right) .
$$

We can get the classical chiral ring relations by substituting $\vec{a}$ by $\mathcal{O}_{n}, n=2, \cdots, N$.

A more elegant derivation of the classical chiral ring relation can be given by expanding the characteristic polynomial of $\Phi$,

$$
\begin{aligned}
\operatorname{det}(x-\Phi) & =x^{N} \exp \operatorname{Tr} \log \left(1-\frac{\Phi}{x}\right) \\
& =x^{N} \exp \left(-\sum_{n=1}^{\infty} \frac{1}{n x^{n}} \operatorname{Tr} \Phi^{n}\right) \\
& =x^{N}-x^{N-2} \mathcal{O}_{2}-x^{N-3} \mathcal{O}_{3}-x^{N-4}\left(\mathcal{O}_{4}-\frac{1}{2} \mathcal{O}_{2,2}\right)+\cdots
\end{aligned}
$$

Since the left-hand side is classically equal to the polynomial $\prod_{\alpha=1}^{N}\left(x-a_{\alpha}\right)$, all the coefficients of negative powers of $x$ in the series expansion must vanish. In this way, we directly obtain the classical chiral ring relations. Notice that the classical chiral ring relations are independent of details of the hypermultiplets. Quantum mechanically, we can take the same set of basis chiral operators, but instanton effects lead to important modifications of the chiral ring relations.

\subsection{Chiral trace relations in the $\Omega$-background}

Since the $\Omega$-background breaks the translational symmetry of $\mathbb{R}^{4}$, we can no longer use the position-independence and the cluster decomposition to reduce the vacuum expectation value of a multi-trace chiral operator to those of single-trace chiral operators. Consequently, we cannot avoid multi-trace chiral operators in our discussion.

A brute force way to derive the chiral trace relations in the $\Omega$-background is as follows. One first computes the vacuum expectation values of chiral operators $\mathcal{O}_{n}$ up to certain high order in (1.3). Then the Coulomb branch parameters $\vec{a}=\left(a_{1}, \cdots, a_{N}\right)$ can be solved in terms of $\mathcal{O}_{n}, n=2, \cdots, N$, with coefficients involving the masses $\vec{m}$, the instanton counting parameter $\mathrm{q}$ and the $\Omega$-deformation parameters $\varepsilon_{1}, \varepsilon_{2}$. Substituting back to the expressions for other chiral operators, we may then guess the chiral trace relations in the $\Omega$-background. In practice, the computation can be extremely tedious, and we would like to look for a better approach.

Inspired by the derivation of the classical chiral ring relations using the characteristic polynomial of $\Phi$, we consider the $y$-observable [29],

$$
y(x)=x^{N} \exp \left(-\sum_{n=1}^{\infty} \frac{1}{n x^{n}} \operatorname{Tr} \Phi^{n}\right)
$$


where we implicitly put $\Phi$ at the origin $0 \in \mathbb{R}^{4}$, which is the fixed point of the $\operatorname{SO}(4)$ rotation symmetry of $\mathbb{R}^{4}$. At the classical level,

$$
y(x)^{\mathrm{cl}}=x^{N} \exp \left(-\sum_{n=1}^{\infty} \frac{1}{n x^{n}} \sum_{\alpha=1}^{N} a_{\alpha}^{n}\right)=\prod_{\alpha=1}^{N}\left(x-a_{\alpha}\right) .
$$

Hence $y(x)$ reduces to the characteristic polynomial det $(x-\Phi)$ classically.

In order to evaluate $y(x)$ at the instanton configuration $\vec{\lambda}$, we use the fact that [10]

$$
\left[\operatorname{Tr} e^{\beta \Phi}\right]_{\vec{\lambda}}=\mathcal{E}_{\vec{\lambda}}=\sum_{\alpha=1}^{N}\left[e^{\beta a_{\alpha}}-P \sum_{\square \in \lambda^{(\alpha)}} e^{\beta\left(a_{\alpha}+c_{\square}\right)}\right] .
$$

Here $\varepsilon_{\vec{\lambda}}$ is the equivariant Chern character of the universal bundle with the universal instanton connection over $\mathbb{C}^{2} \times \mathcal{M}_{N}$ evaluated at the fixed point $0 \times \vec{\lambda}$, where $\mathcal{M}_{N}$ is the moduli space of framed $\mathrm{U}(N)$ instantons on $\mathbb{C}^{2}$. We define

$$
P=\left(1-e^{\beta \varepsilon_{1}}\right)\left(1-e^{\beta \varepsilon_{2}}\right),
$$

and

$$
c_{\square=(i, j)}=\varepsilon_{1}(i-1)+\varepsilon_{2}(j-1) .
$$

We can compute $\left[\operatorname{Tr} \Phi^{n}\right]_{\vec{\lambda}}$ by expanding (2.9) in powers of $\beta$, and then we have

$$
[y(x)]_{\vec{\lambda}}=\prod_{\alpha=1}^{N}\left[\left(x-a_{\alpha}\right) \prod_{\square \in \lambda^{(\alpha)}} \frac{\left(x-a_{\alpha}-c_{\square}-\varepsilon_{1}\right)\left(x-a_{\alpha}-c_{\square}-\varepsilon_{2}\right)}{\left(x-a_{\alpha}-c_{\square}\right)\left(x-a_{\alpha}-c_{\square}-\varepsilon\right)}\right],
$$

where $\varepsilon=\varepsilon_{1}+\varepsilon_{2}$. It is convenient to write $[y(x)]_{\vec{\lambda}}$ succinctly as $[29]$

$$
[y(x)]_{\vec{\lambda}}=\epsilon\left[e^{\beta x} \mathcal{E}_{\vec{\lambda}}^{\vee}\right]=(-1)^{N} \epsilon\left[e^{-\beta x} \mathcal{E}_{\vec{\lambda}}\right],
$$

where $\epsilon$ is the conversion operator which maps characters into weights,

$$
\epsilon\left\{\sum_{i} n_{i} e^{\beta w_{i}}\right\}=\prod_{i} w_{i}^{n_{i}}
$$

and $\vee$ is the dual operator,

$$
\left(\sum_{i} n_{i} e^{\beta w_{i}}\right)^{\vee}=\sum_{i} n_{i} e^{-\beta w_{i}}
$$

After carrying out many pairwise cancelations, $[y(x)]_{\vec{\lambda}}$ becomes

$$
[y(x)]_{\vec{\lambda}}=\prod_{\alpha=1}^{N} \frac{\prod_{\boxplus \in \partial_{+} \lambda^{(\alpha)}}\left(x-a_{\alpha}-c_{\boxplus}\right)}{\prod_{\boxminus \in \partial_{-} \lambda^{(\alpha)}}\left(x-a_{\alpha}-c_{\boxminus}-\varepsilon\right)},
$$

where $\partial_{ \pm} \lambda^{(\alpha)}$ are boxes that can be added to or removed from $\lambda^{(\alpha)}$ while keeping the resulting configuration a well-defined Young diagram. 
Unlike the characteristic polynomial, the function $[y(x)]_{\vec{\lambda}}$ has singularities in $x$. Fortunately, it was found in [29] that the all-instanton information in the $\Omega$-background can be encoded in a system of non-perturbative Dyson-Schwinger equations

$$
\langle X(y(x+\cdots))\rangle=\mathcal{T}(x),
$$

where composite operators $X(x)$ are built out of $y$-observables, and are called qq-characters. $\mathcal{T}(x)$ is a polynomial in $x$. Hence, there is no singularities in $\langle X(x)\rangle$ for finite $x$. Although the $y$-observable only depends on the vector multiplet, the qq-characters contain the information of the hypermultiplet.

Physically, a qq-character in an $\mathcal{N}=2$ supersymmetric gauge theory can be interpreted as the observable obtained by integrating out the auxiliary gauge theory living on a space transverse to the physical spacetime. As shown in [39], the moduli space of framed instantons on the combination of the physical spacetime and the auxiliary transverse space is compact when we turn on the appropriate $\Omega$-deformation and background $B$-field. Consequently, $\langle X(x)\rangle$ cannot have singularities in $x$ since there is no phase transitions or no runaway flat directions at any special value of $x$.

In this paper, we mainly focus on the fundamental qq-character which takes the form

$$
x(x)=y(x)+\cdots .
$$

Here terms in $\cdots$ will cancel the poles of $y(x)$ in the vacuum expectation value. In the weak coupling limit $\mathrm{q} \rightarrow 0$, the fundamental qq-character $X(x) \rightarrow y(x)$. We can follow the same logic of the derivation of the classical chiral ring relations on $\mathbb{R}^{4}$ to directly obtain the exact chiral trace relations in the $\Omega$-background by expanding $X(x)$ around $x=\infty$ and requiring the vacuum expectation values of $x^{-n}$ coefficients $X^{(-n)}$ to be zero for all $n \in \mathbb{Z}^{+}$,

$$
\left\langle x^{(-n)}\right\rangle=0
$$

\section{Basic examples of SU $(N)$ gauge theories}

In this section, we illustrate our general strategy using two examples, the pure $\mathrm{SU}(N)$ gauge theory, and the $\mathrm{SU}(N)$ gauge theory with $2 N$ fundamental hypermultiplets.

\subsection{Pure SU $(N)$ theory}

\subsubsection{Partition function and qq-characters}

The partition function of the pure $\mathrm{SU}(N)$ gauge theory in the $\Omega$-background depends on the instanton counting parameter $\mathrm{q}=\Lambda^{2 N}$, the Coulomb branch parameter $\vec{a}=\left(a_{1}, \cdots, a_{N}\right)$ and the $\Omega$-deformation parameters $\varepsilon_{1}, \varepsilon_{2}$. Applying the supersymmetric localization techniques, the partition function is given by the sum over a collection of $N$ Young diagrams $\vec{\lambda}=\left(\lambda^{(1)}, \cdots, \lambda^{(N)}\right)[4,20,29]$,

$$
\mathcal{Z}\left(\vec{a}, \mathrm{q} ; \varepsilon_{1}, \varepsilon_{2}\right)=\mathrm{q}^{-\frac{1}{2 \varepsilon_{1} \varepsilon_{2}} \sum_{\alpha=1}^{N} a_{\alpha}^{2}} \sum_{\vec{\lambda}} \mathrm{q}^{|\vec{\lambda}|} \mid\left[\frac{\mathcal{E}_{\vec{\lambda}} \mathcal{E}_{\vec{\lambda}}^{\vee}}{P^{\vee}}\right],
$$


where $|\vec{\lambda}|$ is the total number of boxes in the $N$ Young diagrams $\vec{\lambda}$. Therefore, the measure factor is

$$
\mu_{\vec{\lambda}}=\mathrm{q}^{|\vec{\lambda}|-\frac{1}{2 \varepsilon_{1} \varepsilon_{2}} \sum_{\alpha=1}^{N} a_{\alpha}^{2}} \epsilon\left[\frac{\mathcal{E}_{\vec{\lambda}} \mathcal{E}_{\vec{\lambda}}^{\vee}}{P^{\vee}}\right] .
$$

In this simple example, it is easy to find the fundamental qq-character by comparing the measure factors $\mu_{\vec{\lambda}}$ and $\mu_{\vec{\lambda}^{\prime}}$, where $\vec{\lambda}^{\prime}$ is obtained from $\vec{\lambda}$ by removing a box $\mathbf{\square} \in \partial_{-} \lambda^{(\alpha)}$ for certain $\alpha \in\{1, \cdots, N\}$. Using the succinct notations (2.14) and (2.15), we have

$$
\frac{\mu_{\vec{\lambda}^{\prime}}}{\mu_{\vec{\lambda}}}=\left.\mathrm{q}^{\left|\overrightarrow{\lambda^{\prime}}\right|-|\vec{\lambda}|}\right|_{\epsilon}\left[\frac{\mathcal{E}_{\vec{\lambda}^{\prime}} \mathcal{E}_{\vec{\lambda}^{\prime}}^{\vee}-\mathcal{E}_{\vec{\lambda}} \mathcal{E}_{\vec{\lambda}}^{\vee}}{P^{\vee}}\right] .
$$

Since $\vec{\lambda}$ contains one more box than $\vec{\lambda}^{\prime}$, we know that

$$
\left|\vec{\lambda}^{\prime}\right|-|\vec{\lambda}|=-1
$$

Meanwhile, according to (2.9), we have

$$
\mathcal{E}_{\vec{\lambda}^{\prime}}=\mathcal{E}_{\vec{\lambda}}+P \xi, \quad \xi=e^{\beta\left(a_{\alpha}+c \boldsymbol{\varpi}\right)},
$$

which gives

$$
\mathcal{E}_{\vec{\lambda}^{\prime}} \mathcal{E}_{\vec{\lambda}^{\prime}}^{\vee}-\mathcal{E}_{\vec{\lambda}} \mathcal{E}_{\vec{\lambda}}^{\vee}=\mathcal{E}_{\vec{\lambda}^{\prime}} P^{\vee} \xi^{\vee}+\mathcal{E}_{\vec{\lambda}}^{\vee} P \xi
$$

Therefore, we have

$$
\frac{\mu_{\vec{\lambda}^{\prime}}}{\mu_{\vec{\lambda}}}=\mathrm{q}^{-1} \epsilon\left[\mathcal{E}_{\vec{\lambda}^{\prime}} \xi^{\vee}+e^{\beta \varepsilon} \mathcal{E}_{\vec{\lambda}}^{\vee} \xi\right]
$$

Now using (2.13), and the identity

$$
\begin{aligned}
& \operatorname{Res}_{x=a_{\alpha}+c_{\varpi}}[y(x+\varepsilon)]_{\vec{\lambda}} \\
& =\operatorname{Res}_{x=a_{\alpha}+c \boldsymbol{\square}}\left([y(x+\varepsilon)]_{\vec{\lambda}^{\prime}} \frac{\left(x-a_{\alpha}-c \mathbf{\square}+\varepsilon_{1}\right)\left(x-a_{\alpha}-c_{\square}+\varepsilon_{2}\right)}{\left(x-a_{\alpha}-c_{\square}\right)\left(x-a_{\alpha}-c_{\square}+\varepsilon\right)}\right) \\
& =\frac{\varepsilon_{1} \varepsilon_{2}}{\varepsilon}\left[y\left(a_{\alpha}+c \varpi+\varepsilon\right)\right]_{\vec{\lambda}^{\prime}},
\end{aligned}
$$

we get

$$
\operatorname{Res}_{x=a_{\alpha}+c \boldsymbol{\square}}\left([y(x+\varepsilon)]_{\vec{\lambda}} \mu_{\vec{\lambda}}\right)=\operatorname{Res}_{x=a_{\alpha}+c}\left((-1)^{N-1} \mathbf{q}\left[y(x)^{-1}\right]_{\vec{\lambda}^{\prime}} \mu_{\vec{\lambda}^{\prime}}\right) .
$$

As a result, we can take the fundamental qq-character of the theory to be

$$
X(x)=y(x+\varepsilon)+(-1)^{N} \mathrm{q} y(x)^{-1},
$$

whose vacuum expectation value $\langle X(x)\rangle$ has no singularities in $x$, since the residues at all potential poles vanish due to pairwise cancellations. From the large $x$ behavior of $\mathcal{X}(x)$, we know that $\langle X(x)\rangle$ is a polynomial in $x$. 


\subsubsection{Chiral trace relations}

In the following, we shall write down explicitly the chiral trace relations in the $\Omega$-background resulting from $\left\langle X^{(-1)}\right\rangle=\left\langle X^{(-2)}\right\rangle=\left\langle X^{(-3)}\right\rangle=0$ for the gauge group $\mathrm{SU}(2)$ and $\mathrm{SU}(3)$. The generalizations to higher orders or higher gauge groups are straightforward.

We denote

$$
u_{n_{1}, n_{2}, \cdots, n_{\ell}}=\left\langle\mathcal{O}_{n_{1}, n_{2}, \cdots, n_{\ell}}\right\rangle .
$$

It is useful to notice from the structure of the partition function that

$$
\left\langle\mathcal{O}_{2}^{n} \mathcal{O}\right\rangle=\frac{1}{\mathcal{Z}}\left(-\varepsilon_{1} \varepsilon_{2} \mathrm{q} \frac{d}{d \mathrm{q}}\right)^{n}(\langle\mathcal{O}\rangle \mathcal{Z})=\left(u_{2}-\varepsilon_{1} \varepsilon_{2} \mathrm{q} \frac{d}{d \mathrm{q}}\right)^{n}\langle\mathcal{O}\rangle .
$$

In particular, we have

$$
\begin{aligned}
u_{2,2} & =u_{2}^{2}-\varepsilon_{1} \varepsilon_{2} u_{2}^{\prime}, \\
u_{2,2,2} & =u_{2}^{3}-3 \varepsilon_{1} \varepsilon_{2} u_{2}^{\prime} u_{2}+\varepsilon_{1}^{2} \varepsilon_{2}^{2} u_{2}^{\prime \prime},
\end{aligned}
$$

where we denote

$$
u_{n}^{\prime}=\mathrm{q} \frac{d}{d \mathrm{q}} u_{n}, \quad u_{n}^{\prime \prime}=\left(\mathrm{q} \frac{d}{d \mathrm{q}}\right)^{2} u_{n} .
$$

For the $\mathrm{SU}(2)$ theory, we have

$$
\begin{aligned}
& x^{(-1)}=-\mathcal{O}_{3}, \\
& x^{(-2)}=-\mathcal{O}_{4}+\frac{1}{2} \mathcal{O}_{2,2}+\mathrm{q}+\varepsilon \mathcal{O}_{3}, \\
& x^{(-3)}=-\mathcal{O}_{5}+\mathcal{O}_{2,3}+2 \varepsilon\left(\mathcal{O}_{4}-\frac{1}{2} \mathcal{O}_{2,2}\right)-\varepsilon^{2} \mathcal{O}_{3} .
\end{aligned}
$$

The non-perturbative Dyson-Schwinger equations $\left\langle X^{(-1)}\right\rangle=\left\langle X^{(-2)}\right\rangle=\left\langle X^{(-3)}\right\rangle=0$ lead to

$$
\begin{aligned}
& u_{3}=0, \\
& u_{4}=\frac{1}{2}\left(u_{2}^{2}-\varepsilon_{1} \varepsilon_{2} u_{2}^{\prime}\right)+\mathrm{q}, \\
& u_{5}=2 \varepsilon \mathrm{q} .
\end{aligned}
$$

We see that the classical chiral ring relations are modified both by the instanton corrections (charactered by q) and by the $\Omega$-deformation (charactered by $\varepsilon_{1}, \varepsilon_{2}$ ). Our results provide an easy proof of the prediction made in $[23,24]$ where they used the explicit localization results. The unavoidable derivatives, which may seem peculiar in the original approach, appear naturally in our approach.

Repeating the same kind of computation in the case of $\mathrm{SU}(3)$, we find

$$
\begin{aligned}
x^{(-1)}= & -\mathcal{O}_{4}+\frac{1}{2} \mathcal{O}_{2,2}, \\
x^{(-2)}= & -\mathcal{O}_{5}+\mathcal{O}_{2,3}+\varepsilon\left(\mathcal{O}_{4}-\frac{1}{2} \mathcal{O}_{2,2}\right), \\
x^{(-3)}= & -\mathcal{O}_{6}+\mathcal{O}_{2,4}+\frac{1}{2} \mathcal{O}_{3,3}-\frac{1}{6} \mathcal{O}_{2,2,2}-\mathrm{q} \\
& +2 \varepsilon\left(\mathcal{O}_{5}-\mathcal{O}_{2,3}\right)-\varepsilon^{2}\left(\mathcal{O}_{4}-\frac{1}{2} \mathcal{O}_{2,2}\right) .
\end{aligned}
$$


The non-perturbative Dyson-Schwinger equations $\left\langle X^{(-1)}\right\rangle=\left\langle X^{(-2)}\right\rangle=\left\langle X^{(-3)}\right\rangle=0$ lead to

$$
\begin{aligned}
& u_{4}=\frac{1}{2}\left(u_{2}^{2}-\varepsilon_{1} \varepsilon_{2} u_{2}^{\prime}\right), \\
& u_{5}=u_{2} u_{3}-\varepsilon_{1} \varepsilon_{2} u_{3}^{\prime}, \\
& u_{6}=\frac{1}{3}\left(u_{2}^{3}-3 \varepsilon_{1} \varepsilon_{2} u_{2}^{\prime} u_{2}+\varepsilon_{1}^{2} \varepsilon_{2}^{2} u_{2}^{\prime \prime}\right) u_{2}^{3}+\frac{1}{2} u_{3,3}-\mathrm{q} .
\end{aligned}
$$

Notice that in the $\Omega$-background, $u_{3,3}$ cannot be expressed in terms of $u_{2}$ and $u_{3}$. Our result matches the prediction made in [24] perfectly.

\section{$3.2 \mathrm{SU}(N)$ theory with $2 N$ fundamental hypermultiplets}

\subsubsection{Partition function and qq-characters}

The partition function of the $\mathrm{SU}(N)$ gauge theory with $2 N$ fundamental hypermultiplets in the $\Omega$-background is given by [4, 20, 29],

$$
\mathcal{Z}\left(\vec{a}, \vec{m}, \mathrm{q} ; \varepsilon_{1}, \varepsilon_{2}\right)=\mathrm{q}^{-\frac{1}{2 \varepsilon_{1} \varepsilon_{2}} \sum_{\alpha=1}^{N} a_{\alpha}^{2}} \sum_{\vec{\lambda}} \mathrm{q}^{|\vec{\lambda}|} \mid \epsilon\left[\frac{\mathcal{E}_{\vec{\lambda}} \mathcal{E}_{\vec{\lambda}}^{\vee}-\mathcal{M} \mathcal{E}_{\vec{\lambda}}}{P^{\vee}}\right],
$$

which depends on the Coulomb branch parameter $\vec{a}=\left(a_{1}, \cdots, a_{N}\right)$, the masses $\vec{m}=\left(m_{1}, \cdots, m_{2 N}\right)$, the instanton counting parameter $\mathrm{q}=e^{2 \pi \mathbf{i} \tau}$ with $\tau$ being the microscopic complexified coupling constant, and the $\Omega$-deformation parameters $\varepsilon_{1}, \varepsilon_{2}$. Here $\mathcal{M}$ encodes the information of masses

$$
\mathcal{M}=\sum_{f=1}^{2 N} e^{\beta m_{f}}
$$

The qq-character of the theory can be obtained following the same procedure as in the case of pure $\mathrm{SU}(N)$ gauge theory. In particular, we have

$$
\frac{\mu_{\vec{\lambda}^{\prime}}}{\mu_{\vec{\lambda}}}=\mathrm{q}^{-1} \epsilon\left[\mathcal{E}_{\vec{\lambda}^{\prime}} \xi^{\vee}+e^{\beta \varepsilon} \mathcal{E}_{\vec{\lambda}}^{\vee} \xi-e^{\beta \varepsilon} \mathcal{M} \xi\right]
$$

Accordingly, the fundamental qq-character can be taken to be

$$
X(x)=y(x+\varepsilon)+(-1)^{N} \mathrm{q} \mathcal{P}(x) y(x)^{-1},
$$

where

$$
\mathcal{P}(x)=\prod_{f=1}^{2 N}\left(x+m_{f}+\varepsilon\right)=x^{2 N}+\sum_{n=1}^{2 N} x^{2 N-n} M_{n} .
$$

Again, $\langle X(x)\rangle$ is a polynomial in $x$. 


\subsubsection{Chiral trace relations}

Let us consider the gauge group $\mathrm{SU}(2)$. The first few terms of $X^{(-n)}$ are

$$
\begin{aligned}
X^{(-1)}= & -(1-\mathrm{q}) \mathcal{O}_{3}+\mathrm{q} M_{1} \mathcal{O}_{2}+\mathrm{q} M_{3}, \\
X^{(-2)}= & -(1-\mathrm{q}) \mathcal{O}_{4}+\left(\mathrm{q} M_{1}+\varepsilon\right) \mathcal{O}_{3}+\frac{1}{2}(1+\mathrm{q}) \mathcal{O}_{2,2} \\
& +\mathrm{q} M_{2} \mathcal{O}_{2}+\mathrm{q} M_{4} \\
X^{(-3)}= & -(1-\mathrm{q}) \mathcal{O}_{5}+\left(\mathrm{q} M_{1}+2 \varepsilon\right) \mathcal{O}_{4}+(1+\mathrm{q}) \mathcal{O}_{2,3} \\
& +\left(\mathrm{q} M_{2}-\varepsilon^{2}\right) \mathcal{O}_{3}+\left(\frac{1}{2} \mathrm{q} M_{1}-\varepsilon\right) \mathcal{O}_{2,2}+\mathrm{q} M_{3} \mathcal{O}_{2} .
\end{aligned}
$$

Because of the non-perturbative Dyson-Schwinger equations, $\left\langle x^{(-1)}\right\rangle=\left\langle x^{(-2)}\right\rangle=\left\langle x^{(-3)}\right\rangle=0$, we have

$$
\begin{aligned}
u_{3}= & \frac{\mathrm{q}}{1-\mathrm{q}}\left(M_{1} u_{2}+M_{3}\right), \\
u_{4}= & \frac{\mathrm{q} M_{1}+\varepsilon}{1-\mathrm{q}} u_{3}+\frac{1+\mathrm{q}}{2(1-\mathrm{q})}\left(u_{2}^{2}-\varepsilon_{1} \varepsilon_{2} u_{2}^{\prime}\right)+\frac{\mathrm{q}}{1-\mathrm{q}} M_{2} u_{2}+\frac{\mathrm{q}}{1-\mathrm{q}} M_{4} \\
= & \frac{1+\mathrm{q}}{2(1-\mathrm{q})}\left(u_{2}^{2}-\varepsilon_{1} \varepsilon_{2} u_{2}^{\prime}\right)+\left(\frac{\mathrm{q}\left(\mathrm{q} M_{1}+\varepsilon\right) M_{1}}{(1-\mathrm{q})^{2}}+\frac{\mathrm{q} M_{2}}{1-\mathrm{q}}\right) u_{2} \\
& +\frac{\mathrm{q}\left(\mathrm{q} M_{1}+\varepsilon\right) M_{3}}{(1-\mathrm{q})^{2}}+\frac{\mathrm{q}}{1-\mathrm{q}} M_{4}, \\
u_{5}= & \frac{\mathrm{q} M_{1}+2 \varepsilon}{1-\mathrm{q}} u_{4}+\frac{1+\mathrm{q}}{1-\mathrm{q}}\left(u_{2} u_{3}-\varepsilon_{1} \varepsilon_{2} u_{3}^{\prime}\right) \\
& +\frac{\mathrm{q} M_{2}-\varepsilon^{2}}{1-\mathrm{q}} u_{3}+\frac{\mathrm{q} M_{1}-2 \varepsilon}{2(1-\mathrm{q})}\left(u_{2}^{2}-\varepsilon_{1} \varepsilon_{2} u_{2}^{\prime}\right)+\frac{\mathrm{q} M_{3}}{1-\mathrm{q}} u_{2} \\
= & \frac{\mathrm{q}\left(M_{1}+2 \varepsilon\right)}{(1-\mathrm{q})^{2}}\left(u_{2}^{2}-\varepsilon_{1} \varepsilon_{2} u_{2}^{\prime}\right) \\
& +\left(\frac{\mathrm{q}\left(\mathrm{q} M_{1}+\varepsilon\right)\left(\mathrm{q} M_{1}+2 \varepsilon\right) M_{1}}{(1-\mathrm{q})^{3}}+\frac{\mathrm{q}\left(\mathrm{q} M_{1}+2 \varepsilon\right) M_{2}}{(1-\mathrm{q})^{2}}+\frac{\mathrm{q} M_{3}}{1-\mathrm{q}}\right) u_{2} \\
& +\frac{\mathrm{q}^{2}\left(\mathrm{q} M_{1}+\varepsilon\right)\left(\mathrm{q} M_{1}+2 \varepsilon\right) M_{3}}{(1-\mathrm{q})^{3}}+\frac{\mathrm{q}\left(\mathrm{q} M_{1}+2 \varepsilon\right)}{(1-\mathrm{q})^{2}} M_{4} \\
& +\frac{1+\mathrm{q}}{1-\mathrm{q}}\left(u_{2} u_{3}-\varepsilon_{1} \varepsilon_{2} u_{3}^{\prime}\right)+\frac{\mathrm{q} M_{2}-\varepsilon^{2}}{1-\mathrm{q}} u_{3} .
\end{aligned}
$$

Our result confirms the results obtained in [27]. We see that the presence of the hypermultiplet significantly modifies the chiral trace relations in the $\Omega$-background.

\section{The linear quiver gauge theory}

Let us briefly sketch how our method can be generalized to linear quiver gauge theories.

The gauge group of the theory is

$$
G=\prod_{i=1}^{r} \mathrm{SU}(N)_{i} .
$$


For each $\mathrm{SU}(N)_{i}$ factor, we have a vector multiplet transforming in the adjoint representation of $\mathrm{SU}(N)_{i}$. The matter content of the theory spits into $N$ hypermultiplets in the anti-fundamental representation of $\mathrm{SU}(N)_{1}$, one hypermultiplet in the bifundamental representation of $\mathrm{SU}(N)_{i} \times \mathrm{SU}(N)_{i+1}$ for $i=1, \cdots, r-1$, and $N$ fundamental representation of $\mathrm{SU}(N)_{r}$. The action of the theory is parametrized by the instanton counting parameters

$$
\underline{\mathbf{q}}=\left\{\mathrm{q}_{i}=e^{2 \pi \mathrm{i} \tau_{i}}, i=1, \cdots r\right\},
$$

where $\tau_{i}$ is the complexified gauge coupling for $\mathrm{SU}(N)_{i}$. It is convenient to extend the gauge group $\prod_{i=1}^{r} \mathrm{SU}(N)_{i}$ to $\prod_{i=0}^{r+1} \mathrm{U}(N)_{i}$ if we set $\mathrm{q}_{0}=\mathrm{q}_{r+1}=0$. All the masses are formally viewed as Coulomb branch parameters, leading to the extended moduli space with parameters

$$
\left(a_{i, \alpha}\right)_{\alpha=1}^{N}, \quad i=0, \cdots, r+1 .
$$

The physical Coulomb branch parameters are given by

$$
\mathrm{a}_{i, \alpha}=a_{i, \alpha}-\frac{1}{N} \sum_{\alpha=1}^{N} a_{i, \alpha}, \quad i=1, \cdots, r .
$$

We introduce $z_{0}, z_{1}, \cdots, z_{r+1}$, such that $z_{-1}=\infty, z_{r+1}=0$, and

$$
\mathrm{q}_{i}=\frac{z_{i}}{z_{i-1}}, \quad i=1, \cdots, r .
$$

We can define $y$-observables for $i=0$ and $i=r+1$ as

$$
y_{0}(x)=\prod_{\alpha=1}^{N}\left(x-a_{0, \alpha}\right), \quad y_{r+1}(x)=\prod_{\alpha=1}^{N}\left(x-a_{r+1, \alpha}\right) .
$$

The fundamental qq-characters are given by [29]

$$
\begin{aligned}
x_{\ell}(x) & =\frac{y_{0}(x+\varepsilon(1-\ell))}{z_{0} z_{1} \cdots z_{\ell-1}} \sum_{\substack{I \subset[0, r] \\
|I|=\ell}} \prod_{i \in I}\left[z_{i} \Xi_{i}\left(x+\varepsilon\left(h_{I}(i)+1-\ell\right)\right)\right] \\
& =y_{\ell}(x+\varepsilon)+\cdots, \quad \ell=0,1, \cdots, r+1,
\end{aligned}
$$

where $[0, r]=\{0,1,2, \cdots, r\}$, the function $h_{I}(i)$ is the number of elements in the set $I$ which is less than $i$, and

$$
\Xi_{i}(x)=\frac{y_{i+1}(x+\varepsilon)}{y_{i}(x)}, \quad i=0, \cdots, r .
$$

In order to deal with all of the fundamental qq-characters at the same time, we introduce the generating function

$$
\begin{aligned}
G_{r}(x ; t) & =\Delta_{r}^{-1} y_{0}(x)^{-1} \sum_{\ell=0}^{r+1} z_{0} z_{1} \cdots z_{\ell-1} t^{\ell} x_{\ell}(x-\varepsilon(1-\ell)) \\
& =\Delta_{r}^{-1} \sum_{I \subset[0, r]}\left[\left(\prod_{i \in I} t z_{i}\right) \prod_{i \in I} \Xi_{i}\left(x+\varepsilon h_{I}(i)\right)\right],
\end{aligned}
$$


where

$$
\Delta_{r}=\prod_{i=0}^{r}\left(1+t z_{i}\right)=\sum_{I \subset[0, r]}\left(\prod_{i \in I} t z_{i}\right)
$$

is a normalization factor. We can find a relation between $G_{r}(x ; t)$ and $G_{r-1}(x ; t)$ by classifying the set $I \subset[0, r]$ depending on whether $r \in I$,

$$
\begin{aligned}
G_{r}(x ; t)= & \Delta_{r}^{-1} \sum_{I^{\prime} \subset[0, r-1]}\left[\left(\prod_{i \in I^{\prime}} t z_{i}\right) \prod_{i \in I^{\prime}} \Xi_{i}\left(x+\varepsilon h_{I^{\prime}}(i)\right)\right] \\
& +\Delta_{r}^{-1} t z_{r} \sum_{I^{\prime} \subset[0, r-1]}\left[\left(\prod_{i \in I^{\prime}} t z_{i}\right) \Xi_{r}\left(x+\varepsilon\left|I^{\prime}\right|\right) \prod_{i \in I^{\prime}} \Xi_{i}\left(x+\varepsilon h_{I^{\prime}}(i)\right)\right] \\
= & \frac{1}{1+t z_{r}} G_{r-1}(x ; t)+\frac{t z_{r}}{1+t z_{r}} \Delta_{r-1}^{-1} \Xi_{r}\left(x+\varepsilon t \frac{\partial}{\partial t}\right)\left(\Delta_{r-1} G_{r-1}(x ; t)\right) .
\end{aligned}
$$

The large $x$ expansion of the function $\Xi_{i}(x)$ is

$$
\Xi_{i}(x)=\exp \sum_{n=1}^{\infty}\left(\frac{\mathcal{O}_{n}^{(i)}}{x^{n}}-\frac{\mathcal{O}_{n}^{(i+1)}}{(x+\varepsilon)^{n}}\right)=\sum_{n=0}^{\infty} \frac{\zeta_{i, n}}{x^{n}},
$$

where

$$
\mathcal{O}_{n}^{(i)}=\frac{1}{n} \operatorname{Tr} \Phi_{i}^{n}, \quad i=0, \cdots, r+1 .
$$

The first few terms of $\zeta_{i, n}$ are

$$
\begin{aligned}
\zeta_{i, 0}= & 1 \\
\zeta_{i, 1}= & \mathcal{O}_{1}^{(i)}-\mathcal{O}_{1}^{(i+1)}, \\
\zeta_{i, 2}= & \mathcal{O}_{2}^{(i)}-\mathcal{O}_{2}^{(i+1)}+\varepsilon \mathcal{O}_{1}^{(i+1)}+\frac{1}{2}\left(\mathcal{O}_{1}^{(i)}-\mathcal{O}_{1}^{(i+1)}\right)^{2} \\
\zeta_{i, 3}= & \mathcal{O}_{3}^{(i)}-\mathcal{O}_{3}^{(i+1)}+\left(\mathcal{O}_{1}^{(i)}-\mathcal{O}_{1}^{(i+1)}\right)\left(\mathcal{O}_{2}^{(i)}-\mathcal{O}_{2}^{(i+1)}\right) \\
& +\left(\mathcal{O}_{1}^{(i)}-\mathcal{O}_{1}^{(i+1)}\right)^{3}+\varepsilon\left(\mathcal{O}_{1}^{(i)}-\mathcal{O}_{1}^{(i+1)}\right) \mathcal{O}_{1}^{(i+1)} \\
& +2 \varepsilon \mathcal{O}_{2}^{(i+1)}-\varepsilon^{2} \mathcal{O}_{1}^{(i+1)} .
\end{aligned}
$$

We can then expand $G_{r}(x ; t)$ as

$$
G_{r}(x ; t)=\sum_{n=0}^{\infty} \frac{G_{r}^{(n)}(t)}{x^{n}},
$$

and use (4.11) to compute the expansion coefficients $G_{r}^{(n)}(t)$ recursively. The first few terms are given by [31]

$$
\begin{aligned}
G_{r}^{(1)}= & U_{r}[1], \\
G_{r}^{(2)}= & U_{r}[2]+U_{r}[1,1]-\varepsilon U_{r}[0,1], \\
G_{r}^{(3)}= & U_{r}[3]+U_{r}[2,1]+U_{r}[1,2]-\varepsilon\left(U_{r}[1,1]+2 U_{r}[0,2]\right)+\varepsilon^{2} U_{r}[0,1] \\
& +U_{r}[1,1,1]-\varepsilon\left(2 U_{r}[0,1,1]+U_{r}[1,0,1]\right)+2 \varepsilon^{2} U_{r}[0,0,1],
\end{aligned}
$$


where $U_{r}\left[s_{1}, s_{2}, \cdots, s_{\ell}\right]$ is defined for non-negative integers $\left[s_{1}, \cdots, s_{\ell}\right]$ as

$$
U_{r}\left[s_{1}, s_{2}, \cdots, s_{\ell}\right]=\sum_{0 \leq i_{1}<\cdots<i_{\ell} \leq r} \prod_{n=1}^{\ell}\left(\frac{t z_{i_{n}}}{1+t z_{i_{n}}} \zeta_{i_{n}, s_{n}}\right) .
$$

The non-perturbative Dyson-Schwinger equations result in the relations

$$
0=\left\langle\left(y_{0}(x) G_{r}(x ; t)\right)^{(-n)}\right\rangle=\sum_{n=0}^{N} \mathcal{A}_{j}\left\langle G_{r}^{(N+n-j)}(t)\right\rangle, \quad n \in \mathbb{Z}^{+},
$$

where

$$
y_{0}(x)=\prod_{\alpha=1}^{N}\left(x-a_{0, \alpha}\right)=\sum_{j=0}^{N} \mathcal{A}_{j} x^{N-j} .
$$

After expressing $G_{r}^{(n)}(t)$ entirely in terms of chiral operators using (4.14)(4.16)(4.17), we finally derive the chiral trace relations in the $\Omega$-background from (4.18).

\section{Acknowledgments}

We are grateful to Nikita Nekrasov and Wenbin Yan for helpful discussions. SJ and XZ are supported by the US Department of Energy under grant DE-SC0010008. The work of SJ was also supported by the NSF grant PHY 1404446 and by the Simons Center for Geometry and Physics.

Open Access. This article is distributed under the terms of the Creative Commons Attribution License (CC-BY 4.0), which permits any use, distribution and reproduction in any medium, provided the original author(s) and source are credited.

\section{References}

[1] N. Seiberg and E. Witten, Electric-magnetic duality, monopole condensation and confinement in $N=2$ supersymmetric Yang-Mills theory, Nucl. Phys. B 426 (1994) 19 [Erratum ibid. B 430 (1994) 485] [hep-th/9407087] [INSPIRE].

[2] N. Seiberg and E. Witten, Monopoles, duality and chiral symmetry breaking in $N=2$ supersymmetric QCD, Nucl. Phys. B 431 (1994) 484 [hep-th/9408099] [INSPIRE].

[3] G.W. Moore, N. Nekrasov and S. Shatashvili, Integrating over Higgs branches, Commun. Math. Phys. 209 (2000) 97 [hep-th/9712241] [INSPIRE].

[4] N.A. Nekrasov, Seiberg-Witten prepotential from instanton counting, Adv. Theor. Math. Phys. 7 (2003) 831 [hep-th/0206161] [INSPIRE].

[5] N. Nekrasov and A. Okounkov, Seiberg-Witten theory and random partitions, Prog. Math. 244 (2006) 525 [hep-th/0306238] [INSPIRE].

[6] S. Shadchin, Saddle point equations in Seiberg-Witten theory, JHEP 10 (2004) 033 [hep-th/0408066] [INSPIRE].

[7] S. Shadchin, Cubic curves from instanton counting, JHEP 03 (2006) 046 [hep-th/0511132] [INSPIRE]. 
[8] N. Nekrasov and V. Pestun, Seiberg-Witten geometry of four dimensional $N=2$ quiver gauge theories, arXiv:1211.2240 [INSPIRE].

[9] X. Zhang, Seiberg-Witten geometry of four-dimensional $N=2$ SO-USp quiver gauge theories, Phys. Rev. D 100 (2019) 125015 [arXiv:1910.10104] [InSPIRE].

[10] A.S. Losev, A. Marshakov and N.A. Nekrasov, Small instantons, little strings and free fermions, hep-th/0302191 [INSPIRE].

[11] A. Marshakov and N. Nekrasov, Extended Seiberg-Witten Theory and Integrable Hierarchy, JHEP 01 (2007) 104 [hep-th/0612019] [INSPIRE].

[12] N.A. Nekrasov, Two-dimensional topological strings revisited, Lett. Math. Phys. 88 (2009) 207 [INSPIRE].

[13] X. Zhang, Partition function of $\mathcal{N}=2$ supersymmetric gauge theory and two-dimensional Yang-Mills theory, Phys. Rev. D 96 (2017) 025008 [arXiv:1609.09050] [InSPIRE].

[14] D. Gaiotto, $N=2$ dualities, JHEP 08 (2012) 034 [arXiv:0904.2715] [INSPIRE].

[15] D. Gaiotto, G.W. Moore and A. Neitzke, Wall-crossing, Hitchin Systems and the WKB Approximation, arXiv:0907.3987 [INSPIRE].

[16] L.F. Alday, D. Gaiotto and Y. Tachikawa, Liouville Correlation Functions from Four-dimensional Gauge Theories, Lett. Math. Phys. 91 (2010) 167 [arXiv:0906.3219] [INSPIRE].

[17] N. Wyllard, $A(N-1)$ conformal Toda field theory correlation functions from conformal $N=2 \mathrm{SU}(N)$ quiver gauge theories, JHEP 11 (2009) 002 [arXiv:0907.2189] [INSPIRE].

[18] N.A. Nekrasov and S.L. Shatashvili, Quantization of Integrable Systems and Four Dimensional Gauge Theories, in Proceedings, 16th International Congress on Mathematical Physics (ICMP09), Prague, Czech Republic, 3-8 August 2009, pp. 265-289 (2009) [DOI] [arXiv: 0908.4052] [INSPIRE].

[19] N. Nekrasov, A. Rosly and S. Shatashvili, Darboux coordinates, Yang-Yang functional and gauge theory, Nucl. Phys. Proc. Suppl. 216 (2011) 69 [arXiv:1103.3919] [InSPIRE].

[20] N. Nekrasov, V. Pestun and S. Shatashvili, Quantum geometry and quiver gauge theories, Commun. Math. Phys. 357 (2018) 519 [arXiv:1312.6689] [INSPIRE].

[21] F. Cachazo, M.R. Douglas, N. Seiberg and E. Witten, Chiral rings and anomalies in supersymmetric gauge theory, JHEP 12 (2002) 071 [hep-th/0211170] [INSPIRE].

[22] F. Cachazo, N. Seiberg and E. Witten, Chiral rings and phases of supersymmetric gauge theories, JHEP 04 (2003) 018 [hep-th/0303207] [INSPIRE].

[23] M. Beccaria, A. Fachechi and G. Macorini, Chiral trace relations in $\Omega$-deformed $\mathcal{N}=2$ theories, JHEP 05 (2017) 023 [arXiv:1702.01254] [INSPIRE].

[24] A. Fachechi, G. Macorini and M. Beccaria, Chiral trace relations in $\Omega$-deformed $\mathcal{N}=2$ theories, J. Phys. Conf. Ser. 965 (2018) 012013 [inSPIRE].

[25] V.A. Alba, V.A. Fateev, A.V. Litvinov and G.M. Tarnopolskiy, On combinatorial expansion of the conformal blocks arising from AGT conjecture, Lett. Math. Phys. 98 (2011) 33 [arXiv: 1012.1312] [INSPIRE].

[26] V.A. Fateev and A.V. Litvinov, Integrable structure, W-symmetry and AGT relation, JHEP 01 (2012) 051 [arXiv:1109.4042] [INSPIRE]. 
[27] F. Fucito, J.F. Morales and R. Poghossian, Wilson loops and chiral correlators on squashed spheres, JHEP 11 (2015) 064 [arXiv:1507.05426] [INSPIRE].

[28] S.K. Ashok et al., Chiral observables and S-duality in $N=2^{*} \mathrm{U}(N)$ gauge theories, JHEP 11 (2016) 020 [arXiv: 1607.08327] [INSPIRE].

[29] N. Nekrasov, BPS/CFT correspondence: non-perturbative Dyson-Schwinger equations and qq-characters, JHEP 03 (2016) 181 [arXiv: 1512.05388] [INSPIRE].

[30] N. Nekrasov, BPS/CFT correspondence V: BPZ and KZ equations from qq-characters, arXiv: 1711.11582 [INSPIRE].

[31] S. Jeong and X. Zhang, BPZ equations for higher degenerate fields and non-perturbative Dyson-Schwinger equations, arXiv:1710.06970 [INSPIRE].

[32] N. Nekrasov, BPS/CFT correspondence IV: $\sigma$-models and defects in gauge theory, Lett. Math. Phys. 109 (2019) 579 [arXiv:1711.11011] [INSPIRE].

[33] S. Jeong and N. Nekrasov, Opers, surface defects and Yang-Yang functional, arXiv: 1806.08270 [INSPIRE].

[34] S. Jeong, Splitting of surface defect partition functions and integrable systems, Nucl. Phys. B 938 (2019) 775 [arXiv: 1709.04926] [INSPIRE].

[35] J.-E. Bourgine, M. Fukuda, K. Harada, Y. Matsuo and R.-D. Zhu, $(p, q)$-webs of DIM representations, $5 d \mathcal{N}=1$ instanton partition functions and qq-characters, JHEP 11 (2017) 034 [arXiv: 1703.10759] [INSPIRE].

[36] J.-E. Bourgine and S. Jeong, New quantum toroidal algebras from $5 D \mathcal{N}=1$ instantons on orbifolds, arXiv: 1906.01625 [INSPIRE].

[37] V. Pestun, Localization of gauge theory on a four-sphere and supersymmetric Wilson loops, Commun. Math. Phys. 313 (2012) 71 [arXiv:0712.2824] [InSPIRE].

[38] N. Hama and K. Hosomichi, Seiberg-Witten Theories on Ellipsoids, JHEP 09 (2012) 033 [arXiv:1206.6359] [INSPIRE].

[39] N. Nekrasov, BPS/CFT correspondence II: Instantons at crossroads, moduli and compactness theorem, Adv. Theor. Math. Phys. 21 (2017) 503 [arXiv:1608.07272] [INSPIRE]. 